\title{
STRONGLY MINIMAL GROUPS IN THE THEORY OF COMPACT COMPLEX SPACES
}

\author{
MATTHIAS ASCHENBRENNER, RAHIM MOOSA, AND THOMAS SCANLON
}

ABSTRACT. We characterise strongly minimal groups interpretable in elementary extensions of compact complex analytic spaces.

\section{INTRODUCTION}

In [12] Zilber observed that compact complex manifolds may be naturally regarded as structures of finite Morley rank for which the axioms of Zariski-type structures hold. As such, the key to a model theoretic structure theory for sets definable in compact complex manifolds is a description of the interpretable strongly minimal groups. Pillay and Scanlon described these groups in [11] but left open the question of what strongly minimal groups might be definable in elementary extensions of compact complex manifolds. In this paper, we complete the classification.

We regard a compact complex manifold $M$ as a structure in the language having a predicate for each closed analytic subvariety of each Cartesian power of $M$. It is convenient to consider all compact complex analytic spaces at the same time. To do so, we form the many sorted structure $\mathcal{A}$ having a sort for each (isomorphism class of) compact complex analytic space(s) and having as basic relations on the product of sorts $S_{1} \times \cdots \times S_{n}$ the closed analytic subvarieties. As every point in $\mathcal{A}$ is distinguished by a basic relation, this structure cannot be saturated. More seriously, even if one restricts to a single sort $M$ in $\mathcal{A}$ it can happen that there is no countable reduct for which every definable set in $M$ is parametrically definable in the reduct. That is, not all compact complex manifolds are essentially saturated in the sense of [7]. Consequently, to study properties of elementary extensions of $\mathcal{A}$ one cannot work entirely within the standard model.

By definition, elementary properties transfer from $\mathcal{A}$ to its elementary extensions. Much of our work consists of unwinding results for the standard model to find their elementary content. In addition to close readings and reworkings of existing proofs, we use properties of families of analytic spaces. In particular, Grothendieck's relative infinitesimal neighbourhoods play an important role.

The structure $\mathcal{A}$ is interpretable in $\mathbb{R}_{\text {an }}$, the field of real numbers with restricted analytic functions, a well-studied o-minimal structure. We use this observation in only one place in our arguments, but as Peterzil and Starchenko have shown, it could form the basis for a theory of complex analytic spaces over arbitrary ominimal expansions of real closed fields (see $[9,10]$ ). However, the strongly minimal

Date: 8 April 2005.

Matthias Aschenbrenner was partially supported by NSF Grant DMS 03-03618.

Rahim Moosa was partially supported by a University of Waterloo Start-up grant.

Thomas Scanlon was partially supported by an Alfred P. Sloan Fellowship and NSF Grant DMS 03-01771. 
groups definable in the interpreted complex analytic spaces in nonstandard models of the theory of $\mathbb{R}_{\text {an }}$ may have properties not enjoyed by any group in an elementary extension of $\mathcal{A}$. For instance, while every (complex-) one-dimensional group in any elementary extension of $\mathcal{A}$ is algebraic [8], this does not hold in the $\mathbb{R}_{\text {an }}$ world [10]. Thus, while the o-minimal approach may be useful, it must be paired with work internal to $\mathcal{A}$.

Complex algebraic geometry lives in $\mathcal{A}$ in the sense that the field of complex numbers is a definable set (being the complement of the point at infinity on the complex projective line) and the field operations on $\mathbb{C}$ are themselves definable. Moreover, by Chow's theorem, $\mathcal{A}$ induces no additional structure on $\mathbb{C}$. It follows from the classification of locally compact fields that any other field interpretable in $\mathcal{A}$ is definably isomorphic to $\mathbb{C}$. Moosa showed that this conclusion continues to hold in elementary extensions of $\mathcal{A}$ even though the statement of the result is not facially elementary and the Euclidean topology is unavailable in the elementary extensions. Moosa proves in addition a nonstandard version of the Riemann existence theorem from which one may show, using the Zilber trichotomy, that any strongly minimal group interpretable in an elementary extension of $\mathcal{A}$ is either a one-dimensional algebraic group over the (nonstandard) complex numbers or is locally modular.

We direct our attention to the strongly minimal locally modular groups in elementary extensions of $\mathcal{A}$ (though local modularity itself plays no role in our arguments). In $\mathcal{A}$ itself, these groups are the simple nonalgebraic complex tori [11]. We prove a version for elementary extensions.

Theorem 1.1. Suppose $\mathcal{A}^{\prime} \succeq \mathcal{A}$ is a saturated elementary extension of $\mathcal{A}$ with $\mathbb{C}^{\prime}$ its interpretation of $\mathbb{C}$. If $G$ is a strongly minimal group intepretable in $\mathcal{A}^{\prime}$ then either $G$ is definably isomorphic to an algebraic group over $\mathbb{C}^{\prime}$ or $G$ is "compact" in either of the following senses:

(i) viewing $G$ as a definable manifold by interpreting $\mathcal{A}^{\prime}$ in an elementary extension of $\mathbb{R}_{\mathrm{an}}, G$ is definably compact, or

(ii) there exists a Zariski closed set $X$ in $\mathcal{A}^{\prime}$ and a surjective definable map $\pi: X \rightarrow G$ which is holomorphic with respect to the natural nonstandard meromorphic manifold structure on $G$.

Formulation (i) of "compact" is an easy consequence of (ii) and it is in the sense of the latter that the theorem is proved. See Section 4 for a more precise formulation.

We would say that the group $G$ is a nonstandard complex torus if $G$ were of the form $T_{a}$ where $\nu: T \rightarrow B$ is a holomorphic map between compact complex manifolds whose general fibres are (uniformly) complex tori and $a \in B\left(\mathcal{A}^{\prime}\right)$ is a nonstandard point of $B$. It may very well be the case that any "compact" group in the sense of Theorem 1.1 must be a nonstandard complex torus, but we were unable to resolve this question as it implicates some subtle issues in the theory of moduli of complex tori.

This paper is organized as follows. In Section 2 we recall and supply a detailed proof of the main compactification result of [11] that any strongly minimal group $G$ in $\mathcal{A}$ may be embedded as a Zariski open subset of some compact complex manifold. In Section 3 we reformulate the statement of this compactification so as to make its elementary content transparent. In Section 4, we recall some of the theory of elementary extensions of $\mathcal{A}$, show how to transfer part of the compactification theorem and then analyse the case where there is no action of $G$ on its boundary. 
In Section 5 we analyze the remaining case transposing a theorem of Fujiki to the nonstandard context to complete the proof.

Acknowledgements. We are grateful to the Banff International Research Station and the Mathematisches Forschungsinstitut Oberwolfach where part of this work was done during the workshops Interactions between model theory and geometry (BIRS, March 2004) and Model theory and complex analytic geometry (Oberwolfach, July 2004).

\section{Compactifications in $\mathcal{A}$}

In this section we concentrate on proving in detail the following main compactification result from [11].

Proposition 2.1. Let $G$ be a complex manifold expressible as the disjoint union of an open set $U$ and a finite set $F$, where $U$ is a non-empty Zariski open subspace of an irreducible compact complex manifold $X$. There exist:

- a compact complex manifold $G^{*}$ and a holomorphic embedding $\iota: G \rightarrow G^{*}$ such that $\iota(G)$ is a Zariski open subset of $G^{*}$, and

- a holomorphic surjection $\pi: X \rightarrow G^{*}$;

such that the following diagram commutes:

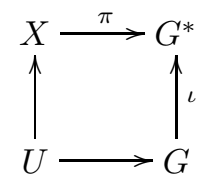

and $\pi$ is a biholomorphism off $\iota(F) \subseteq G^{*}$.

The published proof of this result while correct is, to our taste, incomplete in that the nontrivial verification of the efficacy of the construction is left to the reader. Many of the details of the proof that follows involve routine topological manipulations, but the proof as a whole is remarkably tricky. It should be noted that while we work with the Euclidean topology, the hypotheses and the conclusion of this proposition concern objects definable in the structure $\mathcal{A}$.

We first reduce to the case when $F$ is a singleton. Given $(G, U, X, F)$ we prove the result by induction on $|F|$ with the case of $|F|=0$ being trivial. If $F=F^{\prime} \dot{\cup}\{g\}$, let $G^{\prime}:=G \backslash F^{\prime}$. Then $G^{\prime}$ is a manifold expressible as $U \dot{\cup}\{g\}$. If the result is true for $\left(G^{\prime}, U, X,\{g\}\right)$, then it follows for $(G, U, X, F)$. Indeed, suppose $\iota^{\prime}: G^{\prime} \hookrightarrow$ $\left(G^{\prime}\right)^{*}$ and $\pi^{\prime}: X \rightarrow\left(G^{\prime}\right)^{*}$ satisfy the conclusion for $\left(G^{\prime}, U, X,\{g\}\right)$. After replacing $\left(G^{\prime}\right)^{*}$ by its image under a suitable biholomorphism and composing $\pi^{\prime}$ with this biholomorphism, we may assume that $\left(G^{\prime}\right)^{*}$ contains $G^{\prime}$ as a Zariski open subset and $\iota^{\prime}$ is the natural inclusion $G^{\prime} \subseteq\left(G^{\prime}\right)^{*}$. Now $G$ may be expressed as $G^{\prime} \cup F^{\prime}$, and by induction we find $\iota: G \hookrightarrow G^{*}$ and $\tilde{\pi}:\left(G^{\prime}\right)^{*} \rightarrow G^{*}$ satisfying the conclusion for $\left(G, \iota\left(G^{\prime}\right),\left(G^{\prime}\right)^{*}, F^{\prime}\right)$. Take $\pi:=\widetilde{\pi} \circ \pi^{\prime}: X \rightarrow G^{*}$.

So it suffices to prove the Proposition in the case that $F=\{g\}$ is a singleton, and for the rest of this section we assume that $F$ has this form.

We begin with some notation: For a subset $S$ of a topological space $R$ we denote the closure of $S$ in $R$ by $\mathrm{cl}_{S}(R)$ and the interior of $S$ in $R$ by $\operatorname{int}_{R}(S)$. The boundary of $S$ in $R$ is denoted by bd $\operatorname{bd}_{R}(S):=\operatorname{cl}_{R}(S) \backslash \operatorname{int}_{R}(S)$, and the frontier of $S$ in $R$ by $\operatorname{fr}_{R}(S):=\operatorname{cl}_{R}(S) \backslash S$. When the context is clear we drop the reference to 
$R$ in this notation. Recall also that a subset $S$ of $R$ is called regular open if $S=\operatorname{int}(\operatorname{cl}(S))$. Note that for every $S \subseteq R$, the set $\operatorname{int}(\operatorname{cl}(S))$ is regular open and $\operatorname{cl}(\operatorname{int}(\operatorname{cl}(S))) \subseteq \operatorname{cl}(S)$, with equality if $S$ is open.

Lemma 2.2. Let $V \subseteq G$ be a regular open co-ordinate neighbourhood of $g$ in $G$ and let $D$ be the frontier of $\mathrm{cl}_{G}(V) \backslash\{g\}$ in $X$. That is,

$$
D:=\operatorname{cl}_{X}\left(\operatorname{cl}_{G}(V) \backslash\{g\}\right) \backslash\left(\mathrm{cl}_{G}(V) \backslash\{g\}\right) .
$$

Then $D \cup(V \backslash\{g\})$ is open in $X$.

Indeed, we will show that $D \cup(V \backslash\{g\})=\operatorname{int}_{X}\left(\operatorname{cl}_{X}(V \backslash\{g\})\right)$. Note that $D$ is nonempty as $\mathrm{cl}_{G}(V) \backslash\{g\}$ is not compact, and hence not closed, in $X$. The proof of Lemma 2.2 is preceded by a series of claims.

Claim 2.3. $\operatorname{int}_{G}\left(\operatorname{cl}_{G}(V) \backslash\{g\}\right)=V \backslash\{g\}$

Proof. Since $\operatorname{cl}_{G}(V) \backslash\{g\}=\operatorname{cl}_{G}(V) \cap(G \backslash\{g\})$, the set $G \backslash\{g\}$ is open in $G$, and $V$ is regular open in $G$, we have

$$
\operatorname{int}_{G}\left(\operatorname{cl}_{G}(V) \backslash\{g\}\right)=\operatorname{int}_{G}\left(\operatorname{cl}_{G}(V)\right) \cap \operatorname{int}_{G}(G \backslash\{g\})=V \cap(G \backslash\{g\}),
$$

hence $\operatorname{int}_{G}\left(\operatorname{cl}_{G}(V) \backslash\{g\}\right)=V \backslash\{g\}$ as claimed.

Claim 2.4. $U \cap \operatorname{cl}_{X}(V \backslash\{g\})=\operatorname{cl}_{G}(V) \backslash\{g\}$

Proof. Let $x \in U$, and suppose first that $x \in \operatorname{cl}_{G}(V)$. Then any $G$-neighbourhood of $x$ has a nonempty intersection with $V$. If $N \subseteq X$ is any sufficiently small $X$ neighbourhood of $x$, then $g \notin N$ so that $N$ is also a $G$-neighbourhood of $x$; hence $N \cap(V \backslash\{g\}) \neq \varnothing$. This shows $x \in \operatorname{cl}_{X}(V \backslash\{g\})$. Now suppose $x \notin \mathrm{cl}_{G}(V)$, so there is an open neighbourhood $N$ of $x$ in $G$ with $N \subseteq U$ and $N \cap V=\varnothing$. Then $N$ is also an $X$-neighbourhood of $x$ disjoint from $V \backslash\{g\}$, hence $x \notin \operatorname{cl}_{X}(V \backslash\{g\})$.

Claim 2.5. $U \cap \operatorname{int}_{X}\left(\operatorname{cl}_{X}(V \backslash\{g\})\right)=V \backslash\{g\}$

Proof. Since $U$ is an open subspace of both $G$ and $X$, for every $S \subseteq U$ we have $\operatorname{int}_{G} S=\operatorname{int}_{X} S$. The claim follows from this remark and Claim 2.3 by applying int $_{X}$ on both sides of the equation in Claim 2.4.

Claim 2.6. $\operatorname{cl}_{X}\left(\operatorname{cl}_{G}(V) \backslash\{g\}\right)=\operatorname{cl}_{X}(V \backslash\{g\})$

Proof. Certainly $\operatorname{cl}_{X}\left(\operatorname{cl}_{G}(V) \backslash\{g\}\right) \supseteq \operatorname{cl}_{X}(V \backslash\{g\})$, and the reverse inclusion follows by taking $\mathrm{cl}_{X}$ on both sides of the equation stated in Claim 2.4.

Note that from Claims 2.4 and 2.6 we get $D \subseteq X \backslash U$.

Claim 2.7. $\operatorname{cl}_{X}(V \backslash\{g\})=(V \backslash\{g\}) \cup \operatorname{bd}_{G}(V) \cup D$

Proof. By the definition of $D$ and Claim 2.6, $D \cup\left(\operatorname{cl}_{G}(V) \backslash\{g\}\right)=\operatorname{cl}_{X}(V \backslash\{g\})$. On the other hand, $\mathrm{cl}_{G}(V) \backslash\{g\}=\operatorname{bd}_{G}(V) \cup(V \backslash\{g\})$.

By Claim 2.5 we have $\operatorname{bd}_{G}(V) \cap \operatorname{int}_{X}\left(\operatorname{cl}_{X}(V \backslash\{g\})\right)=\varnothing$; hence by Claim 2.7 we get $\operatorname{int}_{X}\left(\operatorname{cl}_{X}(V \backslash\{g\})\right) \subseteq(V \backslash\{g\}) \cup D$. Our aim now is to show the reverse inclusion and thereby prove Lemma 2.2. We first observe:

Claim 2.8. $\operatorname{bd}_{G}(V) \cup D \supseteq \operatorname{bd}_{X}\left(\operatorname{cl}_{X}(V \backslash\{g\})\right)$ 
Proof. Since $V \backslash\{g\} \subseteq \operatorname{int}_{X}\left(\operatorname{cl}_{X}(V \backslash\{g\})\right)$, Claim 2.7 yields

$$
\begin{array}{r}
\operatorname{bd}_{X}\left(\operatorname{cl}_{X}(V \backslash\{g\})\right)=\operatorname{cl}_{X}(V \backslash\{g\}) \backslash \operatorname{int}_{X}\left(\operatorname{cl}_{X}(V \backslash\{g\})\right) \subseteq \\
\operatorname{cl}_{X}(V \backslash\{g\}) \backslash(V \backslash\{g\}) \subseteq \operatorname{bd}_{G}(V) \cup D .
\end{array}
$$

In the next two claims we will use the fact that any complex manifold admitting a finite atlas where the model spaces are polydiscs (so, in particular, a compact complex manifold) is interpretable in the o-minimal structure $\mathbb{R}_{\mathrm{an}}$, the real field equipped with restricted analytic functions. The sets we have been dealing with (i.e., $V, D$, etc.), though not necessarily definable in $\mathcal{A}$, will "live" definably in $\mathbb{R}_{\text {an }}$ and we will thus have recourse to the o-minimal dimension of these sets.

To make this more precise, consider the disjoint union $M:=G \amalg X$ of the complex manifolds $G$ and $X$, that is, the complex manifold whose underlying topological space is the disjoint union of the topological spaces $G$ and $X$, and whose atlas is the disjoint union of the ones of $G$ and $X$. Then $M$, in fact, admits a finite atlas where the model spaces are polydiscs, which we can assume to contain the co-ordinate chart $V$ of $G$. Hence, $M$ can be identified with a definable $\mathbb{C}$-manifold in $\mathbb{R}_{\text {an }}$ (as defined in Section 3 of [10]) in such a way that $V$ becomes definable in $\mathbb{R}_{\text {an }}$. Moreover, since $U$ is an $\mathbb{R}_{\text {an }}$-definable open subset of both $X$ and $G$ (in both cases it is the complement of an analytic set), and since the identification of the $U$ of $G$ with the $U$ of $X$ is biholomorphic, this identification is also $\mathbb{R}_{\mathrm{an}}$-definable. It follows that $\bar{V}^{G} \backslash\{g\}$ as a subset of $X$ is also definable in $\mathbb{R}_{\text {an }}$, and hence so is $D$.

We will use the following property of o-minimal dimension, which is probably well-known, although we could not locate it in the literature. For this we fix an o-minimal structure $R$.

Fact 2.9. Suppose that $R$ expands a real closed field. Let $M$ be a definable manifold of dimension $m>0$ and $S$ a definable open subset of $M$. Then the definable set $\operatorname{bd}_{M}\left(\operatorname{cl}_{M}(S)\right)$ has local dimension $m-1$ at each point $a \in \operatorname{bd}_{M}\left(\operatorname{cl}_{M}(S)\right)$.

For the proof, we first reduce to the case that $M=R^{m}$, by passing to a chart around $a$ and applying the fact that open cells in $R^{m}$ are definably homeomorphic to $R^{m}$, since $R$ expands a real closed field. The next lemma (valid without this extra assumption on $R$ ) and the equality $\operatorname{bd}(\operatorname{cl}(S))=\operatorname{fr}(\operatorname{int}(\operatorname{cl}(S)))$ then immediately yield the claim.

Lemma 2.10. Let $S$ be a non-empty proper definable regular open subset of $R^{m}$, $m>0$. Then $\operatorname{dim} \operatorname{fr}(S)=m-1$.

In the proof of the lemma we use the following notations and observations. Let $S$ be a subset of $R^{m}, m>0$. For $x \in R$ we put $S_{x}:=\left\{y \in R^{m-1}:(x, y) \in S\right\}$. If $S$ is definable, then each of the following sets is finite:

$$
\begin{aligned}
F_{S} & :=\left\{x \in R: \operatorname{fr}\left(S_{x}\right) \neq \operatorname{fr}(S)_{x}\right\} ; \\
C_{S} & :=\left\{x \in R: \operatorname{cl}\left(S_{x}\right) \neq \operatorname{cl}(S)_{x}\right\} ; \\
I_{S} & :=\left\{x \in R: \operatorname{int}\left(S_{x}\right) \neq \operatorname{int}(S)_{x}\right\} .
\end{aligned}
$$

(We have $C_{S}=F_{S}$, and finiteness of $F_{S}$ is shown in [2], Lemma 4.1.7. Using $\operatorname{int}(A)=R^{n} \backslash \operatorname{cl}\left(R^{n} \backslash A\right)$ for all $n>0$ and $A \subseteq R^{n}$ we see that $I_{S}=C_{R^{m} \backslash S}$, hence $I_{S}$ is also finite.) In particular, we obtain: 
Lemma 2.11. Suppose that $S$ is regular open. If $S$ is definable, then $S_{x}$ is regular open for all but finitely many $x \in R$. Moreover, with $\pi: R^{m} \rightarrow R$ denoting the projection onto the first co-ordinate, the set $S^{\prime}:=\left\{x \in \pi(S): S_{x} \neq R^{m-1}\right\}$ is either empty or infinite.

Proof. For the first statement, just note that the set of $x \in R$ such that $S_{x}$ is not regular open is contained in $C_{S} \cup I_{\mathrm{cl}(S)}$. As for the second statement, if $S^{\prime}$ is finite, then $\operatorname{cl}(S)=\operatorname{cl}(\pi(S)) \times R^{m-1}$, hence $S=\operatorname{int}(\operatorname{cl}(S))=\operatorname{int}(\operatorname{cl}(\pi(S))) \times R^{m-1}$, and therefore $S^{\prime}=\varnothing$.

(In the first statement of the previous lemma we cannot replace "all but finitely many" by "all", as the example $S=$ the union of the two open discs of radius 1 centered at $(0,1)$ and $(0,-1)$ in $R^{2}$ shows.)

Proof of Lemma 2.10. By Theorem 4.1.8 in [2] we have $\operatorname{dim} \operatorname{fr}(S)<\operatorname{dim} S=m$, so it suffices to show that $\operatorname{dim} \operatorname{fr}(S) \geq m-1$. For this, we proceed by induction on $m$. Note that $\operatorname{fr}(S) \neq \varnothing$, since $S \neq \varnothing$ and $S \neq R^{m}$. This yields the claim in the case $m=1$. Suppose $m>1$ and the claim holds with $m$ replaced by $m-1$. The definable subset $\pi(S)$ of $R$ is open and non-empty, and the definable subset $S^{\prime}$ of $\pi(S)$ is either empty or has dimension 1. If $S^{\prime}=\varnothing$, then $S=\pi(S) \times R^{m-1}$, hence $\operatorname{fr}(S)=\operatorname{fr}(\pi(S)) \times R^{m-1}$ and thus $\operatorname{dim} \operatorname{fr}(S) \geq m-1$ as required.

By the remarks above, the set $F$ consisting of all $x \in S^{\prime}$ such that $\operatorname{fr}\left(S_{x}\right) \neq(\operatorname{fr} S)_{x}$ or $S_{x}$ is not regular open is finite, and if $x \in S^{\prime} \backslash F$ then $\operatorname{dim} \operatorname{fr}\left(S_{x}\right) \geq m-2$ by inductive hypothesis. Hence if $\operatorname{dim} S^{\prime}=1$, then also $\operatorname{dim}\left(S^{\prime} \backslash F\right)=1$, and

$$
\operatorname{fr}(S) \cap \pi^{-1}\left(S^{\prime} \backslash F\right)=\bigcup_{x \in S^{\prime} \backslash F}\{x\} \times \operatorname{fr}\left(S_{x}\right)
$$

yields $\operatorname{dim} \operatorname{fr}(S) \geq \operatorname{dim}\left(\operatorname{fr}(S) \cap \pi^{-1}\left(S^{\prime} \backslash F\right)\right) \geq m-1$ by [2], Proposition 4.1.5.

Claim 2.12. If $D \cap \operatorname{bd}_{X}\left(\operatorname{cl}_{X}(V \backslash\{g\})\right) \neq \varnothing$, then the o-minimal dimension of $D$ is at least $2 n-1$ where $n$ is the complex dimension of $X$.

Proof. Suppose $x \in D \cap \operatorname{bd}_{X}\left(\operatorname{cl}_{X}(V \backslash\{g\})\right)$. Since $D \cap U=\varnothing$, we have $x \notin \operatorname{bd}_{G}(V)$. As $\operatorname{bd}_{G}(V)$ is also closed in $X$ there exists an open neighbourhood $P$ of $x$ in $X$ such that $P \cap \operatorname{bd}_{G}(V)=\varnothing$. In $\mathbb{R}_{\mathrm{an}}$, o-minimal dimension for complex analytic sets is twice the complex dimension. Hence the o-minimal dimension of $X$ is $2 n$, and the set $V \backslash\{g\}$ is open in $X$ and definable in $\mathbb{R}_{\mathrm{an}}$. By Fact $2.9, \operatorname{bd}_{X}\left(\operatorname{cl}_{X}(V \backslash\{g\})\right)$ has o-minimal dimension $2 n-1$ everywhere, in particular, at $x$. So, the o-minimal dimension of $P \cap \operatorname{bd}_{X}\left(\operatorname{cl}_{X}(V \backslash\{g\})\right)$ is $2 n-1$. Moreover, by 2.8 and our choice of $P$, we get $P \cap \operatorname{bd}_{X}\left(\operatorname{cl}_{X}(V \backslash\{g\})\right) \subseteq D$.

Claim 2.13. $D \subseteq \operatorname{int}_{X}\left(\mathrm{cl}_{X}(V \backslash\{g\})\right)$

Proof. Since $X \backslash U$ is a proper analytic subset of the irreducible space $X$, the complex dimension of $X \backslash U$ is at most $n-1$, and hence its o-minimal dimension is at most $2 n-2$. By $D \cap U=\varnothing$ and Claim 2.12, $D \cap \operatorname{bd}_{X}\left(\operatorname{cl}_{X}(V \backslash\{g\})\right)=\varnothing$. As $D \subseteq \operatorname{cl}_{X}(V \backslash\{g\})$, we conclude that $D \subseteq \operatorname{int}_{X}\left(\operatorname{cl}_{X}(V \backslash\{g\})\right)$.

Proof of Lemma 2.2. We already know that $\operatorname{int}_{X}\left(\operatorname{cl}_{X}(V \backslash\{g\})\right) \subseteq(V \backslash\{g\}) \cup D$. The reverse inclusion also holds, by Claim 2.13. So the set $D \cup(V \backslash\{g\})=$ $\operatorname{int}_{X}\left(\operatorname{cl}_{X}(V \backslash\{g\})\right)$ is open, as desired. 
Lemma 2.14. As in 2.2, suppose $V \subseteq G$ is a regular open co-ordinate neighbourhood of $g$ in $G$ and $D$ is the frontier of $\operatorname{cl}_{G}(V) \backslash\{g\}$ in $X$. Suppose $N \subseteq V$ is another regular open co-ordinate neighbourhood of $g$ in $G$ and let $D_{N}$ be the frontier of $\operatorname{cl}_{G}(N) \backslash\{g\}$ in $X$. Then $D_{N}=D$.

Proof. By Claims 2.5 and 2.6 we have $D=\operatorname{cl}_{X}(V \backslash\{g\}) \cap(X \backslash U)$, and similarly $D_{N}=\operatorname{cl}_{X}(N \backslash\{g\}) \cap(X \backslash U)$ by applying these claims to $N$ in place of $V$. Now

$$
\begin{aligned}
D & =\operatorname{cl}_{X}(V \backslash\{g\}) \cap(X \backslash U) \\
& =\operatorname{cl}_{X}((V \backslash N) \cup(N \backslash\{g\})) \cap(X \backslash U) \\
& =\left[\operatorname{cl}_{X}(V \backslash N) \cup \operatorname{cl}_{X}(N \backslash\{g\})\right] \cap(X \backslash U) \\
& =\left[\operatorname{cl}_{X}(V \backslash N) \cap(X \backslash U)\right] \cup\left[\operatorname{cl}_{X}(N \backslash\{g\}) \cap(X \backslash U)\right],
\end{aligned}
$$

Since $V \backslash N$ is bounded away from $g$, we have $\operatorname{cl}_{X}(V \backslash N)=\operatorname{cl}_{G}(V \backslash N) \subseteq U$, hence $D=\operatorname{cl}_{X}(N \backslash\{g\}) \cap(X \backslash U)=D_{N}$.

Proof of Proposition 2.1. As mentioned before, it suffices to prove the Proposition in the case that $|F|=1$. Let $F=\{g\}$, and set $V$ and $D$ to be as in Lemma 2.2. Let $W \supseteq V$ be a larger co-ordinate neighbourhood around $g$ in $G$ for which $\operatorname{cl}_{G}(V) \subseteq W$. As a set, we define $G^{*}:=(X \backslash D) \dot{\cup}\{g\}$ and the function $\pi: X \rightarrow G^{*}$ by $\pi(x):=g$ if $x \in D$ and $\pi(x):=x$ otherwise. That is, $G^{*}$ is formed by collapsing $D$ to the point $g$, and $\pi$ is this collapsing map.

We give $G^{*}$ a complex manifold structure by specifying a system of local coordinate neighborhoods about each point in $G^{*}$. In what follows we write $\Delta$ for the standard $\operatorname{dim} G$-unit polydisc. Around $g$, take $O_{g}:=V$ with its co-ordinate function $\phi_{g}: \Delta \rightarrow O_{g}$ as the chart. For $x \in X \backslash D$ find a chart $O_{x} \subseteq X \backslash D$ in the sense of $X$ with co-ordinate function $\psi_{x}: \Delta \rightarrow O_{x}$. If $x \in W$, then we may (and do) choose $O_{x}$ so that it is contained in $W \subseteq G$ and, so, $\psi_{x}$ is also a chart in the sense of $G$ as well. If $x \notin W$, then, in particular, $x \notin \mathrm{cl}_{X}(V \backslash\{g\})$. So, we may (and do) choose $O_{x}$ so that $O_{x} \cap V=\varnothing$.

Consider two distinct points $x \neq y \in G^{*}$. We must show that

$$
\vartheta:=\phi_{y}^{-1} \circ \phi_{x} \uparrow \phi_{x}^{-1}\left(O_{x} \cap O_{y}\right)
$$

is holomorphic. If neither $x$ nor $y$ is equal to $g$, then because $X \backslash D$ is a complex manifold, the transition map $\vartheta$ is a transition map in the sense of $X$ and is therefore holomorphic. So, we may suppose that $x=g \neq y$. If $y \notin W$, then $O_{y} \cap O_{g}=\varnothing$ so that there is nothing to check. If $y \in W$, then $\vartheta$ is a transition map in the sense of $G$ and is holomorphic.

Next we verify that $\pi$ is continuous. Let $a \in X$. If $a \notin D$, then there is a neighbourhood about $a$ on which $\pi$ is the identity, so $\pi$ is clearly continuous at $a$. If $a \in D$, and $N \subseteq V \subseteq G^{*}$ is a regular open co-ordinate neighbourhood of $g=\pi(a)$, then

$$
\pi^{-1}(N)=D \cup(N \backslash\{g\})=D_{N} \cup(N \backslash\{g\})
$$

by Lemma 2.14. The latter is open in $X$ by Lemma 2.2 (applied to $N$ instead of $V)$. That is, $\pi$ is continuous at $a$. So $\pi$ is continuous everywhere.

The map $\pi$ is the identity on $X \backslash D$, and from the choice of co-ordinate neighbourhoods it is clear that it is biholomorphic there. Hence to show that $\pi$ is holomorphic on $X$ it suffices to consider $a \in D$ and $N$ a co-ordinate neighbourhood about $a$ in $X$, and show that $f:=\pi \uparrow N$ is holomorphic. Since $D \subseteq X \backslash U$, 
$A:=N \cap D$ is thin in $N$; it is contained in the analytic set $N \cap(X \backslash U)$. Also, $f$ is holomorphic on $N \backslash A$ and locally bounded everywhere (as it is continuous on $N$ ). By the Riemann extension theorem, $f$ is holomorphic on $N$.

Finally, let $\iota: G \rightarrow G^{*}$ be the inclusion map on the underlying sets (recalling that $U \subseteq X \backslash D)$. It follows from the definitions that $\iota$ is an embedding and that $\pi$ agrees with $\iota$ on $U$. It remains to show that $\iota(G)$ is Zariski open in $G^{*}$. Note that $G^{*} \backslash U=\pi(X \backslash U)$ and hence $U$ is Zariski open in $G^{*}$ (by Remmert's Proper Mapping Theorem). It follows that $\iota(G)=U \cup\{g\}$ is constructible and it suffices to show that $U \cup\{g\}$ is open in $G^{*}$. As $\pi$ is a biholomorphism on $U$ we need only consider the point $g$. But $V$ is an open neighbourhood of $g$ in $G^{*}$ contained in $U \cup\{g\}$.

\section{STANDARD STRONGLY Minimal GROUPS}

Suppose $(G, \mu)$ is a group definable in $\mathcal{A}$. We will mostly deal with strongly minimal, hence abelian, $(G, \mu)$. Therefore, we sometimes also write the group operation $(g, h) \mapsto \mu(g, h): G \times G \rightarrow G$ of $G$ as $\mu(g, h)=g+h$, even if $(G, \mu)$ is not abelian. Suppose, moreover, that we can write $G=U \cup F$ where $U$ is a non-empty Zariski open subset of an irreducible compact complex manifold $X$ and $F$ is a finite set of points disjoint from $U$. For example, when $G$ is strongly minimal we can always write $G$ in this way (by quantifier elimination).

Every group interpretable in $\mathcal{A}$ has the structure of a complex Lie group making it into a connected meromorphic group in the sense of [11]. Indeed, the proof given in [1] of the Weil-Hrushovski theorem for groups interpretable in algebraically closed fields generalises immediately to groups interpretable in $\mathcal{A}$.

So $(G, \mu)$ has the structure of a meromorphic group. In particular, Proposition 2.1 applies to the complex manifold $G$, and for now we identify $G$ with $\iota(G) \subseteq G^{*}$. Moreover, $\mu$ is holomorphic with respect to this manifold structure and extends to a meromorphic map $\mu^{*}: G^{*} \times G^{*} \rightarrow G^{*}$. Let $S:=G^{*} \backslash G$ and let $\Gamma\left(\mu^{*}\right) \subseteq\left(G^{*}\right)^{3}$ denote the graph of $\mu^{*}$.

Fact 3.1 (Lemma 3.3 of [11]). Suppose $S \neq \varnothing$. Then every component of $S$ has codimension 1 in $G^{*}$ and $\mu^{*}$ restricts to a meromorphic map $\mu_{S}^{*}: G^{*} \times S \rightarrow S$. Moreover, for each component $C$ of $S, \mu^{*}$ induces a generic action of $(G, \mu)$ on $C$.

More precisely, for each $g \in G, \Gamma\left(\mu^{*}\right)_{g} \cap(C \times C)$ is the graph of a bimeromorphic map $\mu_{C}^{*}(g,-): C \rightarrow C$; and for $g, h \in G$,

$$
\mu_{C}^{*}(g,-) \circ \mu_{C}^{*}(h,-)=\mu_{C}^{*}(g+h,-)
$$

on a non-empty Zariski open subset of $C$.

In order to transfer the above fact to elementary extensions we would like to remove the reference to the compactification $G^{*}$ (whose existence we cannot a priori establish in the nonstandard case). To this end we formulate the following corollary of Proposition 2.1 and Fact 3.1:

Let $\Gamma(\mu) \subseteq G^{3}$ be the graph of $\mu$, and consider the Zariski closure $\overline{\Gamma(\mu) \cap U^{3}}$ of $\Gamma(\mu) \cap U^{3}$ in $X^{3}$. Since the general fibres over $X^{2}$ under the map $\left(x_{1}, x_{2}, x_{3}\right) \mapsto$ $\left(x_{1}, x_{2}\right)$ are singletons, $\overline{\Gamma(\mu) \cap U^{3}}$ has a unique irreducible component $\Gamma \subseteq X^{3}$ which projects onto $X^{2}$.

Corollary 3.2. Either $G$ is compact - and hence is a complex torus - or $\Gamma$ induces a generic action of $(G, \mu)$ on some component of $X \backslash U$. 
The latter case can be stated more precisely as: for some component $C^{\prime}$ of $X \backslash U$, $\Gamma \cap\left(X \times C^{\prime} \times C^{\prime}\right)$ is the graph of a meromorphic map $\mu^{\prime}: X \times C^{\prime} \rightarrow C^{\prime}$; for each $g \in U, \mu^{\prime}(g,-): C^{\prime} \rightarrow C^{\prime}$ is bimeromorphic; and for $g, h \in U$ such that $g+h \in U$,

$$
\mu^{\prime}(g,-) \circ \mu^{\prime}(h,-)=\mu^{\prime}(g+h,-)
$$

on a non-empty Zariski open subset of $C^{\prime}$.

Proof. If $G=G^{*}$ (that is, if $S=\varnothing$ ) then $G$ is a complex torus. So we assume that $S \neq \varnothing$. Let $\pi: X \rightarrow G^{*}$ be as in Proposition 2.1. Then

$$
X \backslash U=\pi^{-1}(S) \cup \pi^{-1}(F)
$$

and $\pi^{-1}(S)$ is just a copy of $S$ in $X$. In particular, every component of $\pi^{-1}(S)$ has codimension 1 in $X$ and hence is a component of $X \backslash U$. Let $C^{\prime}=\pi^{-1}(C)$ be one such component. By Fact $3.1 \mu^{*}$ induces a generic action of $(G, \mu)$ on $C$. Note that $\pi^{3}(\Gamma)$ agrees with $\Gamma\left(\mu^{*}\right)$ on a non-empty Zariski open subset of $\left(G^{*}\right)^{3}$ and hence $\pi^{3}(\Gamma)=\Gamma\left(\mu^{*}\right)$. Since $\pi^{3}$ is biholomorphic over $\left(G^{*} \backslash F\right)^{3}$, it lifts a generic action to a generic action. That is, $\Gamma$ induces a generic action of $(G, \mu)$ on $C^{\prime}$.

Remark 3.3. The components of $X \backslash U$ split up into those coming from the boundary of $G$ in $G^{*}$ and those that collapse to the finite set $F$. It is on the components of the former type that we have a generic action.

When $G$ is strongly minimal, the latter case of Corollary 3.2 implies that $G$ is a linear algebraic group. Indeed, using the fact that $C^{\prime}$ is of lower dimension one shows that $G$ acts holomorphically on $C^{\prime}$ as the identity (see the proof of Lemma 3.5 in [11]). It then follows from an argument of Fujiki's in [3] that $G$ is linear algebraic. We will mimic this argument in elementary extensions.

But first we deal with the former case of Corollary 3.2 in elementary extensions.

\section{Elementary EXTENSIONS AND THE CASE OF NO BOUNDARY ACTION}

Let $\mathcal{A}^{\prime}$ be a sufficiently saturated elementary extension of $\mathcal{A}$. Definable sets and maps in $\mathcal{A}^{\prime}$ can be understood in terms of uniformly definable families of sets and maps in the standard model $\mathcal{A}$. A systematic discussion of this correspondence is given in section 2 of [8], and we restrict ourselves to only a few remarks here.

First, given an irreducible Zariski closed set $F$ in $\mathcal{A}$ we use $F\left(\mathcal{A}^{\prime}\right)$ to denote its interpretation in the nonstandard model. By a generic point of $F$ we will mean a point $a \in F\left(\mathcal{A}^{\prime}\right)$ that is not contained in $H\left(\mathcal{A}^{\prime}\right)$ for any proper Zariski closed subset $H \subseteq F$. By saturation, every irreducible Zariski closed set has generic points. Conversely, given a sort $X$ of $\mathcal{A}$ and a point $a \in X\left(\mathcal{A}^{\prime}\right)$, the locus of $a$ is the smallest Zariski closed set $F \subseteq X$ such that $a \in F\left(\mathcal{A}^{\prime}\right)$. By noetherianity of the Zariski topology, such a Zariski closed set exists (and is irreducible). Note that a Zariski closed subset $F$ of $X$ is the locus of an element $a \in F\left(\mathcal{A}^{\prime}\right)$ if and only if $a$ is generic in $F$.

If $P$ is a $\varnothing$-definable property of points in $F$, then $P$ holds in some non-empty Zariski open subset of $F$ (we say that $P$ holds for general $x \in F$ ) if and only if it holds for a generic point.

By a Zariski closed set in $\mathcal{A}^{\prime}$ over $s$ we mean a set of the form

$$
G\left(\mathcal{A}^{\prime}\right)_{s}:=\left\{x \in X\left(\mathcal{A}^{\prime}\right):(x, s) \in G\left(\mathcal{A}^{\prime}\right)\right\}
$$

where $X, Y$ are sorts of $\mathcal{A}, G \subseteq X \times Y$ is a Zariski closed subset, and $s \in Y\left(\mathcal{A}^{\prime}\right)$. Taking $S \subseteq Y$ to be the locus of $s$ and restricting the second co-ordinate projection 
map to $F:=G \cap(X \times S)$, we see that $G\left(\mathcal{A}^{\prime}\right)_{s}$ is the fibre of $F \rightarrow S$ over $s$. That is, every Zariski closed set in $\mathcal{A}^{\prime}$ is the fibre of a holomorphic map from a compact complex analytic space to an irreducible compact complex analytic space, over a generic point. This description of Zariski closed sets in $\mathcal{A}^{\prime}$ is more canonical and behaves well with respect to parameters: working over additional parameters in $\mathcal{A}^{\prime}$ corresponds to base change in $\mathcal{A}$.

The Zariski closed sets in $\mathcal{A}^{\prime}$ form the closed sets of a noetherian topology on each sort, and every such set can be written uniquely as an irredundant union of finitely many absolutely irreducible Zariski closed sets - sets that cannot be written as a union of two proper Zariski closed subsets. Absolutely irreducible Zariski closed sets are exactly the generic fibres of fibre spaces, i.e., holomorphic maps between irreducible compact complex spaces whose general fibres are irreducible (see Lemma 2.7 of $[8]$ ).

Suppose $A$ and $B$ are absolutely irreducible Zariski closed sets in $\mathcal{A}^{\prime}$, where $A$ is the fibre of $F \rightarrow S$ over some generic point $s \in S\left(\mathcal{A}^{\prime}\right)$ and $B$ is the fibre of $G \rightarrow T$ over some generic point $t \in T\left(\mathcal{A}^{\prime}\right)$. A map $f: A \rightarrow B$ is holomorphic if there exist

- common base extensions $F_{Z}:=F \times_{S} Z \rightarrow Z$ and $G_{Z}:=G \times_{T} Z \rightarrow Z$;

- an irreducible Zariski closed subset $\Gamma$ of the fibre product $F_{Z} \times{ }_{Z} G_{Z}$; and,

- a point $z \in Z$ which maps to $s$ and $t$ respectively,

such that the graph of $f$ is the fibre of $\Gamma \rightarrow Z$ over $z$. In a similar manner, one can define meromorphic maps between absolutely irreducible Zariski closed sets in $\mathcal{A}^{\prime}$.

One advantage of working in a saturated model, besides the existence of generic points, is homogeneity. In particular, if $F$ is a definable set in $\mathcal{A}^{\prime}$ and $P$ is a set of parameters in $\mathcal{A}^{\prime}$, then $F$ is definable over $P$ if and only if every automorphism of $\mathcal{A}^{\prime}$ that fixes $P$ pointwise fixes $F$ setwise. We use this fact often and abbreviate it to the phrase "by automorphisms".

We now extend the notion of meromorphic group as given in [11] to $\mathcal{A}^{\prime}$ :

Definition 4.1. A meromorphic group in $\mathcal{A}^{\prime}$ (or a nonstandard meromorphic group) is given by the following data: a definable group $(\mathcal{G},+)$ in $\mathcal{A}^{\prime}$ with a finite covering by definable sets $W_{1}, \ldots, W_{n}$; and definable bijections $\phi_{i}: W_{i} \rightarrow U_{i}$ where $U_{i}$ is a non-empty Zariski open subset of an absolutely irreducible Zariski closed set $X_{i}$, for $i=1, \ldots, n$; such that

(i) for each $i \neq j$, the subset $\phi_{i}\left(W_{i} \cap W_{j}\right)$ of $X_{i}$ is Zariski open, and the induced bijection from $\phi_{i}\left(W_{i} \cap W_{j}\right)$ to $\phi_{j}\left(W_{i} \cap W_{j}\right)$ is a biholomorphic map that extends to a meromorphic map from $X_{i}$ to $X_{j}$; and

(ii) for each $i, j, k$, the set

$$
\left\{(x, y) \in U_{i} \times U_{j}: \phi_{i}^{-1}(x)+\phi_{j}^{-1}(y) \in W_{k}\right\}
$$

is Zariski open in $X_{i} \times X_{j}$ and the induced map from this set to $U_{k}$ given by $(x, y) \longmapsto \phi_{k}\left(\phi_{i}^{-1}(x)+\phi_{j}^{-1}(y)\right)$ is holomorphic and extends to a meromorphic map from $X_{i} \times X_{j}$ to $X_{k}$.

We say the meromorphic group $(\mathcal{G},+)$ is defined over a set of parameters $P$ if all the definable sets and maps involved in the above data are over $P$.

Lemma 4.2. Every group interpretable in $\mathcal{A}^{\prime}$ has the structure of a nonstandard meromorphic group. 
Proof. By elimination of imaginaries for $\operatorname{Th}(\mathcal{A})$ (see the appendix of [7]) we need only consider definable groups. As in the standard case, the result now follows by the proof of the Weil-Hrushovski theorem (see [1]).

For the rest of this paper, we let $(\mathcal{G},+)$ be a strongly minimal group definable in $\mathcal{A}^{\prime}$. There exists an irreducible compact complex space $Y$, a uniformly definable family $\left(G \rightarrow Y, \mu: G \times_{Y} G \rightarrow G\right.$ ) of groups over $Y$ (in the standard model), and a generic point $a \in Y\left(\mathcal{A}^{\prime}\right)$, such that $(\mathcal{G},+)=\left(G_{a}, \mu_{a}\right)$. Note that the set $G$ may be merely a definable set (not necessarily Zariski closed), and the maps $G \rightarrow Y$ and $\mu$ merely definable functions (not necessarily holomorphic). By strong minimality of $\mathcal{G}$ and quantifier elimination, there exist

- an irreducible compact complex space over $Y, f: X \rightarrow Y$, whose general fibres are smooth and irreducible;

- a non-empty Zariski open set $U$ of $X$; and

- a compact complex space $F$ over $Y$, whose general fibres are finite;

such that $G_{y}=U_{y} \cup F_{y}$ for general $y \in Y$. In particular, $G_{y}$ is a (standard) definable group of the form discussed in the previous section. Note that $\mathcal{G}=U_{a} \cup F_{a}$.

By Lemma $4.2,(\mathcal{G},+)$ is a nonstandard meromorphic group. We can choose $Y$ and $a \in Y\left(\mathcal{A}^{\prime}\right)$ such that this meromorphic group structure is also over $a$. It follows that there is a uniformly definable meromorphic group structure on $\left(G_{y}, \mu_{y}\right)$ for general $y \in Y$.

Proposition 4.3. Suppose that + does not extend to a generic action of $\mathcal{G}$ on any absolutely irreducible components of $X_{a} \backslash U_{a}$. Then for general $y \in Y(\mathcal{A}), G_{y}$ is definably isomorphic to a complex torus.

Moreover, possibly after base extension, there is a definable map $\pi: X \rightarrow G$ over $Y$ such that for general $y \in Y, \pi_{y}: X_{y} \rightarrow G_{y}$ is a holomorphic surjection with respect to the complex manifold structure on $G_{y}$ viewed as a meromorphic group.

That is, $\pi_{a}: X_{a} \rightarrow \mathcal{G}$ is a holomorphic surjection with respect to the nonstandard meromorphic manifold structure on $\mathcal{G}$.

Proof. Let $\Gamma_{a}$ be the unique absolutely irreducible component of $\overline{\Gamma(+) \cap U_{a}^{3}} \subseteq X_{a}^{3}$ that projects onto the first two factors. By automorphisms, $\Gamma_{a}$ is defined over $a$ and hence $\Gamma_{a}$ is the generic fibre over $Y$ of an irreducible analytic subset $\Gamma \subseteq X^{3}$.

Claim 4.4. For generic $g \in X_{a}, \Gamma_{a}(g):=\Gamma_{a} \cap\left(\{g\} \times X_{a}^{2}\right)$ is absolutely irreducible.

Proof of claim. In the standard model the general fibres of of the first projection $\Gamma \rightarrow X$ have a unique maximal dimensional irreducible component that projects onto $X$ (viewing the fibres as subsets of $X \times_{Y} X$ and taking the first projection). This is because they are generically one-to-one over $X$. These distinguished components are uniformly definable over $X$ (by automorphisms). So there is a constructible $\Gamma^{\prime} \subseteq \Gamma$ whose general fibres over $X$ are these distinguished irreducible components of the fibres of $\Gamma$. Counting dimension and using the irreducibility of $\Gamma$ we see that the Zariski closure of $\Gamma^{\prime}$ must be $\Gamma$. As the general fibres of $\Gamma^{\prime}$ are Zariski closed, $\overline{\Gamma^{\prime}}$ and $\Gamma^{\prime}$ have the same general fibres. So the general fibres of $\Gamma$ are irreducible. So the generic fibre is absolutely irreducible.

Possibly after base extension, we may assume that the absolutely irreducible components of $X_{a} \backslash U_{a}$ are defined over $a$ - write them as $\left(C_{1}\right)_{a}, \ldots,\left(C_{n}\right)_{a}$ where $C_{1}, \ldots, C_{n}$ are irreducible analytic subsets of $X$. Now fix $i \leq n$. To say that "+ extends to a generic action of $\mathcal{G}$ on $\left(C_{i}\right)_{a}$ " is to say that 
(i) over some Zariski open subset of $X_{a} \times\left(C_{i}\right)_{a}, \Gamma_{a} \cap\left(X_{a} \times\left(C_{i}\right)_{a} \times\left(C_{i}\right)_{a}\right)$ is the graph of a well-defined function to $\left(C_{i}\right)_{a}$; and

(ii) for generic $g \in X_{a}, \Gamma_{a}^{i}(g):=\Gamma_{a} \cap\left(\{g\} \times\left(C_{i}\right)_{a}^{2}\right)$ induces a well-defined bijection between Zariski open subsets of $\left(C_{i}\right)_{a}$; and

(iii) for generic $g, h \in X_{a}, \Gamma_{a}^{i}(g+h)$ agrees with

$$
\left\{(x, y) \in\left(C_{i}\right)_{a}^{2}: \exists z(x, z) \in \Gamma_{a}^{i}(h) \text { and }(z, y) \in \Gamma_{a}^{i}(g)\right\} .
$$

Both (i) and (ii) are definable properties of $a$ : (i) is expressed by stating that the co-ordinate projection

$$
\Gamma_{a} \cap\left(X_{a} \times\left(C_{i}\right)_{a} \times\left(C_{i}\right)_{a}\right) \rightarrow X_{a} \times\left(C_{i}\right)_{a}
$$

is surjective with generic fibre a singleton; and (ii) is expressed by saying that for generic $g \in X_{a}$, both co-ordinate projections from $\Gamma_{a}^{i}(g):=\Gamma_{a} \cap\left(\{g\} \times\left(C_{i}\right)_{a}^{2}\right)$ to $\left(C_{i}\right)_{a}$ are surjective with generic fibres singletons. (To say that the generic fibre has a property $P$ is equivalent to saying that the set of point in the base over which the fibre has property $P$ is of the same dimension as the base - and hence is definable if $P$ is.) Note that (iii) is always true since $\Gamma_{a}(g+h)$ agrees with

$$
\left\{(x, y) \in X_{a}^{2}: \exists z(x, z) \in \Gamma_{a}(h) \text { and }(z, y) \in \Gamma_{a}(h)\right\}
$$

on the nonempty Zariski open subset of $X_{a}^{2}$ where $\Gamma$ agrees with + ; and hence on all of $X_{a}^{2}$.

It follows that " + does not extend to a generic action of $\mathcal{G}$ on any absolutely irreducible components of $X_{a} \backslash U_{a}$ " is a definable property of $a$. Hence for general $y \in Y, \mu_{y}$ does not extend to a generic action of $G_{y}$ on any irreducible component of $X_{y} \backslash U_{y}$. By Corollary 3.2 this implies that $G_{y}$ is definably isomorphic to a complex torus, as desired.

For the "moreover" clause, fix a sufficiently general $y \in Y$. Let $\iota: G_{y} \rightarrow G_{y}^{*}$ be the compactification of $G_{y}$ obtained in Proposition 2.1. In this case $\iota\left(G_{y}\right)=G_{y}^{*}$, and we set $\pi_{y}: X_{y} \rightarrow G_{y}$ to be the composition of the associated holomorphic surjection $X_{y} \rightarrow G_{y}^{*}$ with $\iota^{-1}$. As $\iota$ is an isomorphism on the complex manifold $G_{y}$ viewed as a meromorphic group, $\pi_{y}$ is a holomorphic surjection with respect to this structure. Despite the optimistic notation, we have yet to verify that $\pi_{y}$ is uniformly definable in $y$ (we do not claim that $\iota$ is). We know from Proposition 2.1 that $\pi_{y}$ is the identity on $U_{y}$, and so it remains to consider $\pi_{y}$ on $X_{y} \backslash U_{y}$. Note that

$$
X_{y} \backslash U_{y}=\pi_{y}^{-1}\left(F_{y}\right)=\bigcup_{f \in F_{y}} \pi_{y}^{-1}(f),
$$

and each $\pi_{y}^{-1}(f)$ is a finite union of components of $X_{y} \backslash U_{y}$. Since $F_{y}$ and the components of $X_{y} \backslash U_{y}$ are uniformly definable in $y$, there are only finitely many possibilities for what $\pi_{y}$ can do on $X_{y} \backslash U_{y}$, and these possibilities can be described uniformly. It follows that one of these possibilities holds on a nonempty Zariski open subset of $Y$, as desired.

Remark 4.5. Proposition 4.3 says that if + does not extend to a generic action on the boundary than $\mathcal{G}$ is "compact" in the sense of Theorem 1.1.

Question 4.6. Does it follow from the conclusion of Proposition 4.3 that (possibly after base extension) there exists a compact complex space $G^{*}$ over $Y$ and a definable map $\iota: G \rightarrow G^{*}$ over $Y$ such that for general $y \in Y, \iota_{y}$ is a definable 
isomorphism between $G_{y}$ and $G_{y}^{*}$ ? If so it would follow that $\mathcal{G}$ is a nonstandard complex torus.

\section{The CASE OF A BOUNDARY ACTION}

In this final section we complete the proof of Theorem 1.1 by dealing with the case when the hypothesis of Proposition 4.3 fails - that is, when + does extend to a generic action of $\mathcal{G}$ on some absolutely irreducible component of $X_{a} \backslash U_{a}$. We show that in this case $(\mathcal{G},+)$ is definably isomorphic to a linear algebraic group over $\mathbb{C}^{\prime}$.

We continue to use the notation established in the previous section. Let $\Gamma_{a}$ be as in the proof of Proposition 4.3; namely the (unique) absolutely irreducible component of $\overline{\Gamma(+) \cap U_{a}^{3}} \subseteq X_{a}^{3}$ that projects onto the first two factors. So $\Gamma_{a}$ is the generic fibre over $Y$ of an irreducible Zariski closed $\Gamma \subseteq X \times_{Y} X \times_{Y} X$. For general $y \in Y$ and general $g \in X_{y}, \Gamma_{y}(g):=\Gamma_{y} \cap\left(\{g\} \times X_{y}^{2}\right)$ (viewed as a subset of $\left.X_{y}^{2}\right)$ is the graph of a meromorphic map $\tau_{g}: X_{y} \rightarrow X_{y}$ which agrees with translation by $g$ on a non-empty Zariski open set.

We now assume that $\tau_{a}$ extends to a generic action of $\mathcal{G}$ on some component $C_{a}$ of $X_{a} \backslash U_{a}$. That is, $\Gamma_{a} \cap\left(X_{a} \times C_{a}^{2}\right)$ contains a unique absolutely irreducible component that projects onto the first two factors - denote it by $\Gamma_{a}^{C}$ - which is the graph of a generic action of $\mathcal{G}$ on $C_{a}$.

Lemma 5.1. $\Gamma_{a}^{C}=X_{a} \times \Delta$ where $\Delta$ denotes the diagonal in $C_{a}^{2}$. That is, $\tau_{a}$ extends trivially to $C_{a}$.

Proof. This is just as in Lemma 3.5 of [11]. Note that the generic types of $\mathcal{G}$ and $C_{a}$ (over $a$ ) are orthogonal. Indeed, by strong minimality of $\mathcal{G}$ any nonorthogonality would be witnessed by (model-theoretic) algebraicity. But $\operatorname{dim}(\mathcal{G})=\operatorname{dim} X_{a}>$ $\operatorname{dim} C_{a}$. Let $g, h$ be generic independent elements of $\mathcal{G}$ and let $x \in C_{a}$ be generic over $\{g, h\}$. Then $\tau_{a}(g, x) \in C_{a}$ is generic in $C_{a}$ over $x$. Hence, each of $g$ and $h$ is independent from $\tau_{a}(g, x)$ over $x$ by orthogonality. As $\mathcal{G}$ has a unique generic type over $\left\{x, \tau_{a}(g, x)\right\}$, we have $\tau_{a}(g, x)=\tau_{a}(h, x)$ and thus $\tau_{a}(g-h, x)=x$. Since $(g-h, x)$ is a generic of $X_{a} \times C_{a}$, we have shown that $\Gamma_{a}^{C}$ agrees with $X_{a} \times \Delta$ on a non-empty Zariski open subset of $X_{a} \times C_{a}^{2}$ - and hence everywhere by absolute irreducibility.

Before continuing we discuss relative automorphism groups from Fujiki [5].

5.1. Relative Automorphism Groups. Suppose $f: Z \rightarrow T$ is proper surjective morphism of irreducible complex analytic spaces (not necessarily reduced or compact). Suppose moreover, that $f$ is flat. We denote by $\operatorname{Aut}(Z / T)$ the group of biholomorphic maps from $Z$ to itself over $T$. As described by Fujiki in [5], $\operatorname{Aut}(Z / T)$ can be endowed with the structure of a reduced analytic space over $T$ by viewing it as Zariski open subset of the relative Douady space of $Z \times_{T} Z$ over $T$, $D\left(Z \times_{T} Z / T\right) \rightarrow T$. The relative Douady space $D\left(Z \times_{T} Z / T\right) \rightarrow T$ parametrises the flat families of analytic subsets of the fibres of $Z \times_{T} Z \rightarrow T$, and the identification of $\operatorname{Aut}(Z / T)$ with a subspace of $D\left(Z \times_{T} Z / T\right)$ is obtained by taking the codes of the graphs of the biholomorphisms (this requires the flatness of $f$ ). Under this identification, $\operatorname{Aut}(Z / T) \rightarrow T$ becomes a complex Lie group over $T$.

Now drop the assumption that $f$ is flat but add the assumptions that $Z$ is compact (though not necessarily reduced) and $T$ is compact and reduced. Then there is a non-empty Zariski open set $U \subseteq T$ such that the restriction $Z_{U} \rightarrow U$ is 
flat. Following [5] we let $\operatorname{Aut}^{*}(Z / T)$ be those irreducible components of the closure of $\operatorname{Aut}\left(Z_{U} / U\right)$ in $D\left(Z \times_{T} Z / T\right)$ that map surjectively onto $T$. It is not hard to see that $\operatorname{Aut}^{*}(Z / T)$ is independent of the choice of $U$. Moreover, shrinking $U$ if necessary, we may assume that:

- $\operatorname{Aut}^{*}(Z / T)$ is the Zariski closure of $\operatorname{Aut}\left(Z_{U} / U\right)$. In fact, for all $t \in U$, $\operatorname{Aut}^{*}(Z / T)_{t}$ is the Zariski closure of $\operatorname{Aut}\left(Z_{U} / U\right)_{t}$.

- If $\nu: \operatorname{Aut}\left(Z_{U} / U\right) \times_{T} \operatorname{Aut}\left(Z_{U} / U\right) \rightarrow \operatorname{Aut}\left(X_{U} / U\right)$ denotes the group operation on $\operatorname{Aut}\left(Z_{U} / U\right)$ over $T$, then $\nu$ extends to a meromorphic map $\nu^{*}: \operatorname{Aut}^{*}(Z / T) \times_{T} \operatorname{Aut}^{*}(Z / T) \rightarrow \operatorname{Aut}^{*}(X / T)$ over $T$.

- If $\sigma_{U}: \operatorname{Aut}\left(Z_{U} / U\right) \times_{T} Z_{U} \rightarrow Z_{U}$ denotes the natural action of $\operatorname{Aut}\left(Z_{U} / U\right)$ on $Z_{U}$ over $T$, then $\sigma_{U}$ is holomorphic and extends to a meromorphic map $\sigma^{*}: \operatorname{Aut}^{*}(Z / T) \times_{T} Z \rightarrow Z$ over $T$.

It is not necessarily the case that $\operatorname{Aut}^{*}(Z / T)$ is compact. It will be compact if $f: Z \rightarrow T$ is Moishezon, or more generally of Kähler-type. This is because under such an assumption the components of the relative Douady spaces are compact (due to Fujiki). We obtain:

Fact 5.2. Suppose $Z$ is an irreducible compact complex space, $T$ is an irreducible and reduced compact complex space, and $f: Z \rightarrow T$ is a holomorphic surjection of Kähler-type. Then for some non-empty Zariski open set $U \subseteq T$, Aut $\left(Z_{U} / U\right) \rightarrow U$ is a definable group over $U$ and its natural action on $Z_{U}$ over $U$ is definable.

Remark 5.3. It will be important to us that $\operatorname{Aut}\left(Z_{U} / U\right)$ is a definable group over $U$ even in the case when $Z$ itself is nonreduced.

Returning to our situation, let $p \in C_{a}$ be a generic point. Possibly after base extension we may assume that $p=\rho(a)$, where $\rho: Y \rightarrow X$ is a holomoprhic section to $f: X \rightarrow Y$.

Let $\Delta_{X / Y}^{(n)}$ denote the $n$th infinitesimal neighbourhood of $X$ over $Y$. That is, the underlying space of $\Delta_{X / Y}^{(n)}$ is $X$ and the structure sheaf is $\mathcal{O}_{X \times_{Y} X} / \mathcal{I}^{(n+1)}$, where $\mathcal{I}$ is the ideal sheaf of the diagonal in $X \times_{Y} X$. An exposition of this construction and various of its properties can be found in [6].

The first co-ordinate projection induces a map $\Delta_{X / Y}^{(n)} \rightarrow X$, whose fibres are described as follows (Proposition 3.3 of [6]): for each $x \in X,\left(\Delta_{X / Y}^{(n)}\right)_{x}$ is canonically isomorphic to the complex space

$$
\left(\{x\}, \mathcal{O}_{X_{f(x)}, x} / \mathfrak{m}_{X_{f(x)}, x}^{n+1}\right),
$$

where $\mathfrak{m}_{X_{f(x)}, x}$ is the maximal ideal of $X_{f(x)}$ at $x$. This complex space is the $n t h$ infinitesimal neighbourhood of $x$ in $X_{f(x)}$ and is denoted by $\Delta_{X_{f(x)}, x}^{(n)}$.

We can restrict $\Delta_{X / Y}^{(n)} \rightarrow X$ to $\rho(Y) \subseteq X$ to obtain a subspace $D^{(n)} \subseteq \Delta_{X / Y}^{(n)}$ such that for each $y \in Y, D_{y}^{(n)}$ is canonically isomorphic to $\Delta_{X_{y}, \rho(y)}^{(n)}$ the $n$th infinitesimal neighbourhood of $\rho(y)$ in $X_{y}$. So $D^{(n)}$ is a Zariski closed analytic subspace of $X$ that projects onto $Y$. Note that $D^{(n)} \rightarrow Y$ is finite and hence projective. In particular it is of Kähler-type, and so Fact 5.2 applies. That is, for $W \subseteq Y$ a sufficiently small non-empty Zariski open subset, $\operatorname{Aut}\left(\left.D^{(n)}\right|_{W} / W\right) \rightarrow W$ is a definable group over $W$ with compactification $\operatorname{Aut}^{*}\left(D^{(n)} / Y\right) \rightarrow Y$. 
Lemma 5.4. For $a \in Y\left(\mathcal{A}^{\prime}\right)$ generic, $\operatorname{Aut}\left(\left.D^{(n)}\right|_{W} / W\right)_{a}$ is definably isomorphic to a linear algebraic group in $\mathcal{A}^{\prime}$.

Proof. Note that for each $y \in W(\mathcal{A})$, Aut $\left(\left.D^{(n)}\right|_{W} / W\right)_{y}=\operatorname{Aut}\left(\left(D^{(n)}\right)_{y}\right)$ is definably isomorphic to a linear algebraic group - the question is how to obtain this uniformly. But $\operatorname{Aut}^{*}\left(D^{(n)} / Y\right) \rightarrow Y$ is projective as $D^{(n)} \rightarrow Y$ is finite and the relative Douady space of a projective map is again projective over the base (see Lemma 5 of [4]). So possibly after base change we can embed $\operatorname{Aut}^{*}\left(D^{(n)} / Y\right)$ into $Y \times \mathbb{P}_{m}$ over $Y$ (see Proposition 4.4 of [8]). Stable embeddability and essential saturation of the projective sort now imply that the generic fibre of $\operatorname{Aut}\left(\left.D^{(n)}\right|_{W} / W\right) \rightarrow W$ is definably isomorphic to a linear algebraic group.

The goal now is to show that for each $n$ there is a natural generically definable map $X_{a} \rightarrow \operatorname{Aut}^{*}\left(D^{(n)} / Y\right)_{a}$. We produce this map as follows: Let

$$
\Gamma^{[n]}:=\Gamma \cap\left(X \times_{Y} D^{(n)} \times_{Y} D^{(n)}\right) .
$$

We mean here of course the sheaf-theoretic intersection. That is, $\Gamma^{[n]}$ is the (nonreduced) closed subspace of $X \times_{Y} D^{(n)} \times_{Y} D^{(n)}$ obtained as the inverse image of $\Gamma$ under the closed embedding of $X \times_{Y} D^{(n)} \times_{Y} D^{(n)}$ in $X \times_{Y} X \times_{Y} X$. For each $y \in Y$ we view

$$
\Gamma_{y}^{[n]}=\Gamma_{y} \cap\left(X_{y} \times D_{y}^{(n)} \times D_{y}^{(n)}\right)
$$

as a family of analytic subspaces of $D_{y}^{(n)} \times D_{y}^{(n)}$ via the first projection map $\Gamma_{y}^{[n]} \rightarrow$ $X_{y}$. We claim that for general $y \in Y$ and general $g \in X_{y}$, the fibre of $\Gamma_{y}^{[n]} \rightarrow X_{y}$ over $g, \Gamma_{y}^{[n]}(g)$, is the graph of an automorphism of $D_{y}^{(n)}$. To make this precise, recall that the graph of a holomorphic map $\phi: A \rightarrow B$ between (possibly non-reduced) complex spaces is the fibre product of $\phi: A \rightarrow B$ and $\operatorname{id}_{B}: B \rightarrow B$. We use the following Lemma.

Lemma 5.5. Suppose $A$ and $B$ are complex analytic spaces, $\Phi \subseteq A \times B$ is an analytic suspace, and $a \in A$ is such that $\Phi$ defines a holomorphic map $\phi$ on a neighbourhood of $a$. Let $b=\phi(a)$ and $\Phi_{(a, b)}^{[n]}:=\Phi \cap\left(\Delta_{A, a}^{(n)} \times \Delta_{B, b}^{(n)}\right)$. Then $\Phi_{(a, b)}^{[n]}$ is the graph of a holomorphic map from $\Delta_{A, a}^{(n)}$ to $\Delta_{B, b}^{(n)}$. Indeed, it is the graph of the restriction of $\phi$ to $\Delta_{A, a}^{(n)}$.

Proof. Note that if $D$ is an open neighbourhood of $a$ in $A$ then the $n$th infinitesimal neighbourhood of $a$ in $D$ coincides with the $n$th infinitesimal neighbourhood of $a$ in $A$. That is, the entire question is local and we may assume that $\Phi$ defines a holomorphic map $\phi$ on all of $A$.

By functoriality of the infinitesimal neighbourhoods, there is a (unique) map

$$
\phi_{(a, b)}^{(n)}: \Delta_{A, a}^{(n)} \rightarrow \Delta_{B, b}^{(n)}
$$

which commutes with the inclusions $\Delta_{A, a}^{(n)} \subseteq A$ and $\Delta_{B, b}^{(n)} \subseteq B$ via $\phi: A \rightarrow B$ (see the discussion on page 3 of [6], for example). So $\phi_{(a . b)}^{(n)}$ is the restriction of $\phi$ to $\Delta_{A, a}^{(n)}$. The graph of $\phi_{(a, b)}^{(n)}$ is the inverse image of the graph of $\phi$ under the closed embedding of $\Delta_{A, a}^{(n)} \times \Delta_{B, b}^{(n)}$ in $A \times B$. That is, the graph of $\phi_{(a, b)}^{(n)}$ is $\Phi_{(a, b)}^{[n]}$.

Corollary 5.6. For general $y \in Y$ and general $g \in X_{y}, \Gamma_{y}^{[n]}(g)$ is the graph of the automorphism of $D_{y}^{(n)}$ induced by $\tau_{g}$. 
Proof. Recall the following facts:

- $\Gamma_{y}(g) \subseteq X_{y}^{2}$ is the graph of the meromorphic map $\tau_{g}: X_{y} \rightarrow X_{y}$,

- $\tau_{g}$ agrees with translation by $g$ on a non-empty Zariski open set,

- $\tau_{g}$ is defined at and fixes $\rho(y) \in C_{y}$ (by Lemma 5.1),

- $\tau_{g}$ is holomorphic in a neighbourhood of $\rho(y)$ - in fact, as $\rho(y)$ is generic in $C_{y}$, the complement of all the components of $X_{y} \backslash U_{y}$ excepting $C_{y}$ is a Zariski-neighbourhood of $\rho(y)$ on which $\tau_{g}$ is holomorphic.

By Lemma 5.5, $\left(\Gamma_{y}(g)\right)_{(\rho(y), \rho(y))}^{[n]}$ is the graph of the holomorphic map $\left(\tau_{g}\right)_{(\rho(y), \rho(y))}^{(n)}$ from $D_{y}^{(n)}$ to itself induced by $\tau_{g}$. On the other hand, $\tau_{g}$ has $\tau_{-g}$ as an inverse on a non-empty Zariski open set, and hence everywhere where they define a holomorphic map - including at $\rho(y)$. By functoriality, $\left(\tau_{g}\right)_{(\rho(y), \rho(y))}^{(n)}$ and $\left(\tau_{-g}\right)_{(\rho(y), \rho(y))}^{(n)}$ are inverses, and so $\left(\Gamma_{y}(g)\right)_{(\rho(y), \rho(y))}^{[n]}$ is the graph of an automorphism of $D_{y}^{(n)}$. Finally, observe that

$$
\left(\Gamma_{y}(g)\right)_{(\rho(y), \rho(y))}^{[n]}=\Gamma_{y}(g) \cap\left(D_{y}^{(n)} \times D_{y}^{(n)}\right)=\Gamma_{y}^{[n]}(g) .
$$

We are in the following situation:

$$
\Gamma^{[n]} \subseteq X \times_{Y}\left(D^{(n)} \times_{Y} D^{(n)}\right)
$$

defines a family of analytic subspaces of $D^{(n)} \times_{Y} D^{(n)}$ parameterised by $X$ over $Y$. By the universal property of relative Douady spaces (together with an application of Hironaka's Flattening Theorem) there is a meromorphic map $\gamma_{n}$ from $X$ to the relative Douady space of $D^{(n)} \times_{Y} D^{(n)}$ over $Y$, which for general $y \in Y$ and general $g \in X_{y}$, takes $g$ to the Douady point of $\Gamma_{y}^{[n]}(g)$. By Corollary 5.6, we have $\gamma_{n}: X \rightarrow \operatorname{Aut}^{*}\left(D^{(n)} / Y\right)$. Moreover this map is generically a group homomorphism:

Lemma 5.7. For general $y \in Y$ and general $g, h \in X_{y}, \gamma_{n}(g+h)=\gamma_{n}(g) \circ \gamma_{n}(h)$.

Proof. As we have a generic action, we know that $\tau_{g+h}=\tau_{g} \circ \tau_{h}$ as meromorphic maps from $X_{y}$ to itself. But by (the proof of) Corollary 5.6, $\gamma(g)$ is the automorphsim $\left(\tau_{g}\right)_{(\rho(y), \rho(y))}^{(n)}$ of $D_{y}^{(n)}$ - and similarly for $h$ and $g+h$. By functoriality of the infinitesimal neighbourhoods,

$$
\left(\tau_{g}\right)_{(\rho(y), \rho(y))}^{(n)} \circ\left(\tau_{h}\right)_{(\rho(y), \rho(y))}^{(n)}=\left(\tau_{g+h}\right)_{(\rho(y), \rho(y))}^{(n)} .
$$

Lemma 5.8. Suppose $y \in Y$ and $g, h \in X_{y}$ are very general. If $\gamma_{n}(g)=\gamma_{n}(h)$ for all $n \in \mathbb{N}$, then $g=h$.

Proof. By 'very general' we will mean outside a countable union of proper Zariski closed sets. Choose $y$ and $g, h$ sufficiently 'general' so as to ensure that for each $n, \gamma_{n}(g)$ and $\gamma_{n}(h)$ are (the Douady points of the graphs of) $\left(\tau_{g}\right)_{(\rho(y), \rho(y))}^{(n)}$ and $\left(\tau_{h}\right)_{(\rho(y), \rho(y))}^{(n)}$ respectively. So $\left(\tau_{g}\right)_{(\rho(y), \rho(y))}^{(n)}=\left(\tau_{h}\right)_{(\rho(y), \rho(y))}^{(n)}$ for all $n$. That is, for each $n$, the restrictions of $\tau_{g}$ and $\tau_{h}$ coincide on the $n$th infinitesimal neighbourhood of $\rho(y)$ in $X_{y}, D_{y}^{(n)}$. Recalling that $D_{y}^{(n)}$ is canonically isomorphic to $\left(\{\rho(y)\}, \mathcal{O}_{X_{y}, \rho(y)} / \mathfrak{m}_{X_{y}, \rho(y)}^{n+1}\right)$, and that $\bigcap_{n} \mathfrak{m}_{X_{y}, \rho(y)}^{n+1}=0$, we conclude that $\tau_{g}=\tau_{h}$ on some local open neighbourhood of $\rho(y)$. As they are meromorphic maps, this means 
that $\tau_{g}=\tau_{h}$ everywhere where they are defined. Since they agree with translation by $g$ and $h$ (respectively) on a non-empty Zariski open set, $g=h$.

Corollary 5.9. For some $N, \gamma_{N}$ is a bimeromorphism with its image.

Proof. We will show that for some $N, \gamma_{N}$ is injective off a countable union of proper Zariski closed sets (this will suffice). Moreover, it suffices to do this fibrewise over $Y$; so fix a sufficently general $y \in Y$. Now let $\Sigma(g, h)$ be the partial type that says $g$ and $h$ are outside the appropriate countable union of Zariski closed sets that makes Lemma 5.8 work, that $\gamma_{n}(g)=\gamma_{n}(h)$ for all $n \in \mathbb{N}$, and that $g \neq h$. By Lemma 5.8 , this type is not realised. By $\omega_{1}$-compactness, as $\Sigma$ is countable, some finite fragment of $\Sigma$ is not realised. Let $N$ be the maximum of the $n$ that appear in such a fragment. This $N$ works (noting that if $m \leq n$ and $\gamma_{n}(g)=\gamma_{n}(h)$ then $\left.\gamma_{m}(g)=\gamma_{m}(h)\right)$.

It follows that in the elementary extension, $\left(\gamma_{N}\right)_{a}: X_{a} \rightarrow \operatorname{Aut}^{*}\left(D^{(n)} / Y\right)_{a}$ gives a definable embedding of a generic subset of $\mathcal{G}$ into $\operatorname{Aut}\left(\left.D^{(n)}\right|_{W} / W\right)_{a}$. The latter is a linear algebraic group (over the corresponding algebraically closed field $\mathbb{C}^{\prime}$ ) by Lemma 5.4. In particular, $\mathcal{G}$ is internal to the projective sort, and hence $(\mathcal{G},+)$ is definably isomorphic to a (strongly minimal) algebraic group. Hence it is either an elliptic curve or a one-dimensional linear algebraic group. But it cannot be an elliptic curve since by Lemma $5.7\left(\gamma_{N}\right)_{a}$ is generically a group embedding into a linear algebraic group. We obtain

Proposition 5.10. Suppose that + does extend to a generic action of $\mathcal{G}$ on some absolutely irreducible components of $X_{a} \backslash U_{a}$. Then $\mathcal{G}$ is definably isomorphic to the multiplicative or additive group of $\mathbb{C}^{\prime}$.

Propositions 4.3 and 5.10 prove Theorem 1.1.

\section{REFERENCES}

[1] E. Bouscaren. Model theoretic versions of Weil's theorem on pregroups. In A. Nesin and A. Pillay, editors, The model theory of groups (Notre Dame, IN, 1985-1987), volume 11 of Notre Dame Math. Lectures, pages 177-185. University of Notre Dame Press, 1989.

[2] L.van den Dries. Tame Topology and O-Minimal Structures, volume 248 of London Math. Soc. Lecture Note Series. University Press, 1998.

[3] A. Fujiki. On automorphism groups of compact Kähler manifolds. Inventiones Mathematicae, 44(3):225-258, 1978.

[4] A. Fujiki. On the Douady space of a compact complex space in the category $\mathcal{C}$. Nagoya Mathematical Journal, 85:189-211, 1982.

[5] A. Fujiki. On a holomorphic fibre bundle with meromorphic structure. Publications of the Research Institute for Mathemtaical Sciences, 19(1):117-134, 1983.

[6] R. Moosa. Jet spaces in complex analytic geometry: An exposition. E-print available at http://arxiv.org/abs/math.LO/0405563.

[7] R. Moosa. On saturation and the model theory of compact Kähler manifolds. To appear in the Journal für die reine und angewandte Mathematik, 2003.

[8] R. Moosa. A nonstandard Riemann existence theorem. Transactions of the American Mathematical Society, 356(5):1781-1797, 2004.

[9] Y. Peterzil and S. Starchenko. Expansions of algebraically closed fields in o-minimal structures. Selecta Math. (N.S.), 7(3):409-445, 2001.

[10] Y. Peterzil and S. Starchenko. Expansions of algebraically closed fields. II. Functions of several variables. Journal of Mathematical Logic, 3(1):1-35, 2003.

[11] A. Pillay and T. Scanlon. Meromorphic groups. Transactions of the American Mathematical Society, 355(10):3843-3859, 2003. 
[12] B. Zilber. Model theory and algebraic geometry. In Proceedings of the 10th Easter Conference on Model Theory, Berlin, 1993.

E-mail address: maschenb@math.uic.edu

University of Illinois, Chicago, Department of Mathematics, Statistics, and ComPuter Science, 851 S. Morgan St. (M/C 249), Chicago, IL 60607-7045, USA

E-mail address: rmoosa@math.uwaterloo.ca

University of Waterloo, Department of Pure Mathematics, 200 University Avenue West, Waterloo, Ontario N2L 3G1, Canada

E-mail address: scanlon@math.berkeley.edu

University of California, Berkeley, Department of Mathematics, Evans Hall, BerkeLEY, CA 94720-3480, USA 